\title{
Improving Discriminative Sequential Learning with Rare-but-Important Associations
}

\author{
Xuan-Hieu Phan \\ Japan Advanced Inst. of Science \& Technology \\ 1-1, Asahidai, Nomi, Ishikawa, 923-1292 Japan \\ hieuxuan@jaist.ac.jp \\ Tu-Bao Ho \\ Japan Advanced Inst. of Science \& Technology \\ 1-1, Asahidai, Nomi, Ishikawa, 923-1292 Japan \\ bao@jaist.ac.jp
}

\begin{abstract}
Discriminative sequential learning models like Conditional Random Fields (CRFs) have achieved significant success in several areas such as natural language processing or information extraction. Their key advantage is the ability to capture various non-independent and overlapping features of inputs. However, several unexpected pitfalls have a negative influence on the model's performance; these mainly come from an imbalance among classes/labels, irregular phenomena, and potential ambiguity in the training data. This paper presents a data-driven approach that can deal with such hard-to-predict data instances by discovering and emphasizing rare-but-important associations of statistics hidden in the training data. Mined associations are then incorporated into these models to deal with difficult examples. Experimental results of English phrase chunking and named entity recognition using CRFs show a significant improvement in accuracy. In addition to the technical perspective, our approach also highlights a potential connection between association mining and statistical learning by offering an alternative strategy to enhance learning performance with interesting and useful patterns discovered from large dataset.
\end{abstract}

\section{Categories and Subject Descriptors}

I.2.6 [Artificial Intelligence]: Learning; H.4 [Information Systems Applications]: Miscellaneous

\section{General Terms}

Algorithms, Experimentation

\section{Keywords}

Discriminative sequential learning, feature selection, information extraction, text segmentation, association rule

Permission to make digital or hard copies of all or part of this work for personal or classroom use is granted without fee provided that copies are not made or distributed for profit or commercial advantage and that copies bear this notice and the full citation on the first page. To copy otherwise, to republish, to post on servers or to redistribute to lists, requires prior specific permission and/or a fee.

KDD'05, August 21-24, 2005, Chicago, Illinois, USA.

Copyright 2005 ACM 1-59593-135-X/05/0008 ...\$5.00.

\author{
Le-Minh Nguyen \\ Japan Advanced Inst. of Science \& Technology \\ 1-1, Asahidai, Nomi, Ishikawa, 923-1292 Japan \\ nguyenml@jaist.ac.jp \\ Susumu Horiguchi \\ Tohoku University \\ Aoba 6-3-09 Sendai, 980-8579 Japan \\ susumu@ecei.tohoku.ac.jp
}

\section{INTRODUCTION}

Conditionally-trained or discriminative models like Maximum Entropy (MaxEnt) [3], Discriminative HMMs [5], Maximum Entropy Markov Models (MEMMs) [18], and CRFs [13] have achieved significant success in many (sequential) labeling and segmenting tasks, such as part-of-speech (POS) tagging [24], text segmentation or shallow parsing [20, 25], information extraction $[8,22]$, object detection in computer vision [26], image analysis and labeling [10, 12], and biological sequence modeling [27]. The noticeable advantage of these models is their flexibility to integrate a variety of arbitrary, overlapping, and non-independent features at different levels of granularity from the observed data.

However, applications employing these models with fixed and hand-built feature templates usually generate a huge number of features, up to millions, e.g., in [25]. This is because one usually utilizes complex templates including conjunctions of atomic context predicates, e.g., n-gram of words or POS tags, to cover as many combinations of statistics as possible without eliminating irrelevant ones. As a result, models using long and fixed conjunction templates are heavily overfitting and time-consuming to train because they contains many teacher-specific and redundant features. To reduce these drawbacks, McCallum [19] proposed a likelihooddriven feature induction for CRFs that is based on a famous feature inducing strategy for exponential models [21]. This method iteratively adds the conjunctions of atomic observational tests that most increase conditional log-likelihood into the model until some stopping criteria are reached. In spite of attaining a trade-off between the number of used features and model accuracy, this strategy may ignore rare but sensitive conjunctions with smaller likelihood gains that are still critical to model performance. Also, when the number of atomic context predicates is large, the number of conjunctions becomes explosive; and thus ranking all conjunctions by likelihood gain is very expensive.

In this paper, we propose a data-driven approach that can identify and emphasize rare-but-important associations or co-occurrences of statistics ${ }^{1}$ hidden the training data to

\footnotetext{
${ }^{1}$ In this paper, terms like "(atomic) context predicates", "(singleton) statistics", or "(atomic) observational tests" are used interchangeably to refer to particular kinds of contextual information observed from the training data
} 
improve prediction accuracy for hard-to-classify instances. The main motivation and the underlying idea of this approach are based on the fact that (sequential) data, such as natural language or biological information, potentially contain the following phenomena that should be the major sources of prediction errors:

- Ambiguous data instances usually contain unclear contextual clues that may result in misleading predictions. For instance, it is quite difficult for a phrase chunker to determine whether the word plans in the text the trip plans for Japan is a singular verb or a plural noun.

- Irregular instances are recognized as exceptions to the common statistics or decisions. For example, a POS tagger may mark walk as a noun in the sentence The disabled walk very slowly because of a regular sequential dependency that a noun should go after an adjective. However, the correct interpretation is that The disabled (i.e., The disabled people) is the subject and walk is a plural verb rather than a noun.

- Unbalanced data occurs when the distribution of classes in the training data is unbalanced. For example, the number of English noun phrases (NP) is much larger than that of other phrase types, e.g., adjective phrases (ADJP). This may lead to low prediction accuracy for minor classes due to the dominance of major ones.

- Frequently-observed vs. less-observed data instances: For instance, a named entity recognizer may identify New York University as a location while it is in fact an organization. This is because New York is observed more frequently than New York University.

- Long dependencies in sequence data: several kinds of sequential training data contain long dependencies among observations at different positions in a sequence. The problem is that one can not always use a sliding window large enough to capture such useful clues because it would generate too many irrelevant features.

Data instances falling into the above situations should be hard examples. Thus, the prediction of their labels does not usually obey the frequently observed statistics. In other words, the simple aggregation of singleton context predicates may lead to misleading predictions because the common statistics always overwhelm uncommon ones. To overcome this pitfall, a model should rely on rare-but-important associations or conjunctions of singleton context predicates to win the dominance of common decisions. In the first example, most contextual supports surrounding plans (e.g., trip is a singular noun, plans ends with $s$ ) tend to say that plans is a singular verb rather than a part of a noun phrase. It is, however, quite easy for the model to recognize plans as a plural noun if relying on an important association like "if the word after plans for is capitalized, then plans should be a plural noun". This association rule emphasizes a rare but important co-occurrence of three factors: plans, for, and the next word is initially capitalized (such as a location like a city or a country). Although such kind of associations may only occur several times in a whole dataset, their appearance is an important source of evidence to deal with difficult instances.
In spite of their benefit, mining all rare-but-important associations of singleton statistics in big datasets is challenging because the number of candidates is prohibitively large. Fortunately, we find that association rule mining techniques, such as FP-growth [11], are very useful for discovering such patterns. In our method, the set of rare-but-important associations is a special subset of rare but highly confident association rules discovered in the training data. Selected associations are then integrated into the learning process according to one of three ways to improve the prediction accuracy for hard instances: (a) associations as normal features, (b) associations as normal features with weighted feature values, and (c) associations as constraints for the inference process.

Derived from a reasonable assumption about rare-butimportant associations and the robustness of association rule mining techniques, our approach offers the following distinctive characteristics: (1) rare-but-important associations are globally discovered from a huge number of candidates with any length and any combination of singleton statistics; (2) models with those associations can deal with difficult instances while preventing overfitting by avoiding long and fixed conjunction templates; (3) users can choose a suitable way to incorporate selected associations into their models. Particularly, 100\%-confidence associations can be integrated into the model in terms of constraints for inference; and (4) our method can be used to improve any discriminative sequential learning application, especially for highly ambiguous and imbalanced data; (5) finally, our work also highlights a potential connection between pattern mining and statistical learning from large datasets.

The rest of this paper is organized as follows. Section 2 briefly introduces linear-chain CRFs, a typical sequential learning model. Section 3 presents the proposed approach. Section 4 describes and discusses the experimental results. Section 5 reviews related work. Finally, conclusions are given in Section 6 .

\section{DISCRIMINATIVE SEQUENTIAL LEARNING}

The goal of labeling/tagging for sequential data is to learn to map observation sequences to their corresponding label sequences, e.g., the sequence of POS tags for words in a sentence. Discriminative HMMs [5], MEMMs [18], and CRFs [13] were intentionally designed for such sequential learning applications. In contrast to generative models like HMMs [23], these models are discriminative, i.e., trained to predict the most likely label sequence given the observation sequence. In this paper, CRFs are referred to as the undirected linear-chain of model states, i.e., conditionally-trained finite state machines (FSMs) that obey the first-order Markov independence assumption. The strength of CRFs is that they can combine both the sequential property of HMMs and the philosophy of MaxEnt as well as global normalization that can avoid the label-bias problem [13]. In our work, CRFs were used to conduct all experiments.

\subsection{Conditional Random Fields}

Let $\mathbf{o}=\left(o_{1}, o_{2}, \ldots, o_{T}\right)$ be some observed data sequence. Let $\mathcal{S}$ be a set of FSM states, each of which is associated with a label, $l \in \mathcal{L}$. Let $\mathbf{s}=\left(s_{1}, s_{2}, \ldots, s_{T}\right)$ be some state sequence, CRFs [13] define the conditional probability of a 
state sequence given an observation sequence as

$$
p_{\theta}(\mathbf{s} \mid \mathbf{o})=\frac{1}{Z(\mathbf{o})} \exp \left[\sum_{t=1}^{T} \sum_{k} \lambda_{k} f_{k}\left(s_{t-1}, s_{t}, \mathbf{o}, t\right)\right],
$$

where $Z(\mathbf{o})=\sum_{\mathbf{s}}, \exp \left(\sum_{t=1}^{T} \sum_{k} \lambda_{k} f_{k}\left(s_{t-1}^{\prime}, s_{t}^{\prime}, \mathbf{o}, t\right)\right)$ is a normalization summing over all label sequences. $f_{k}$ denotes a feature function in the language of maximum entropy modeling and $\lambda_{k}$ is a learned weight associated with feature $f_{k}$. Each $f_{k}$ is either a per-state or a transition feature:

$$
\begin{aligned}
f_{k}^{(\text {per-state })}\left(s_{t}, \mathbf{o}, t\right) & =\delta\left(s_{t}, l\right) x_{k}(\mathbf{o}, t), \\
f_{k}^{(\text {transition })}\left(s_{t-1}, s_{t}, t\right) & =\delta\left(s_{t-1}, l^{\prime}\right) \delta\left(s_{t}, l\right),
\end{aligned}
$$

where $\delta$ denotes the Kronecker- $\delta$. A per-state feature (2) combines the label $l$ of current state $s_{t}$ and a context predicate, i.e., the binary function $x_{k}(\mathbf{o}, t)$ that captures a particular property of the observation sequence $\mathbf{o}$ at time position $t$. For example, the current label is JJ (adjective) and the current word is "sequential". A transition feature (3) represents sequential dependencies by combining the label $l^{\prime}$ of the previous state $s_{t-1}$ and the label $l$ of the current state $s_{t}$, such as the previous label $l^{\prime}=\mathrm{JJ}$ and the current label $l=\mathrm{NN}$ (noun).

\subsection{Inference in CRFs}

Inference in CRFs is to find the most likely state sequence $\mathbf{s}^{*}$ given the observation sequence $\mathbf{o}$,

$$
\begin{aligned}
\mathbf{s}^{*} & =\operatorname{argmax}_{\mathbf{s}} p_{\theta}(\mathbf{s} \mid \mathbf{o}) \\
& =\operatorname{argmax}_{\mathbf{S}}\left\{\exp \left[\sum_{t=1}^{T} \sum_{k} \lambda_{k} f_{k}\left(s_{t-1}, s_{t}, \mathbf{o}, t\right)\right]\right\}
\end{aligned}
$$

In order to find $\mathbf{s}^{*}$, one can apply a dynamic programming technique with a slightly modified version of the original Viterbi algorithm for HMMs [23]. To avoid an exponentialtime search over all possible settings of $\mathbf{s}$, Viterbi stores the probability of the most likely path up to time $t$ which accounts for the first $t$ observations and ends in state $s_{i}$. We denote this probability to be $\varphi_{t}\left(s_{i}\right)(0 \leq t \leq T-1)$ and $\varphi_{0}\left(s_{i}\right)$ to be the probability of starting in each state $s_{i}$. The recursion is given by:

$$
\varphi_{t+1}\left(s_{i}\right)=\max _{s_{j}}\left\{\varphi_{t}\left(s_{j}\right) \exp \left[\sum_{k} \lambda_{k} f_{k}\left(s_{j}, s_{i}, \mathbf{o}, t\right)\right]\right\}
$$

The recursion terminates when $t=T-1$ and the biggest unnormalized probability is $p^{*}=\operatorname{argmax}_{i}\left[\varphi_{T}\left(s_{i}\right)\right]$. At this time, we can backtrack through the stored information to find the most likely sequence $\mathbf{s}^{*}$.

\subsection{Training CRFs}

CRFs are trained by setting the set of weights $\theta=\left\{\lambda_{1}, \ldots\right\}$ to maximize the log-likelihood, $L$, of a given training data set $\mathcal{D}=\left\{\left(\mathbf{o}^{(k)}, \mathbf{l}^{(k)}\right)\right\}_{k=1}^{N}$ :

$$
L=\sum_{j=1}^{N} \log \left(p_{\theta}\left(\mathbf{l}^{(j)} \mid \mathbf{o}^{(j)}\right)\right)-\sum_{k} \frac{\lambda_{k}^{2}}{2 \sigma^{2}}
$$

where the second sum is a Gaussian prior over parameters with variance $\sigma^{2}$, which provides smoothing to deal with sparsity in the training data [4].

When the labels make the state sequence unambiguous, the likelihood function in exponential models such as CRFs is convex, thus searching the global optimum is guaranteed [19]. However, the optimum can not be found analytically. Parameter estimation for CRFs requires an iterative procedure. It has been shown that quasi-Newton methods, such as L-BFGS [15], are most efficient [17, 25]. This method can avoid the explicit estimation of the Hessian matrix of the log-likelihood by building up an approximation of it using successive evaluations of the gradient.

L-BFGS is a limited-memory quasi-Newton procedure for unconstrained optimization that requires the value and gradient vector of the function to be optimized. Let $s_{j}$ denote the state path of training instance $j$ in training set $D$, then the log-likelihood gradient component of $\lambda_{k}$ is

$$
\begin{aligned}
\frac{\delta L}{\delta \lambda_{k}}= & {\left[\sum_{j=1}^{N} C_{k}\left(\mathbf{s}^{(j)}, \mathbf{o}^{(j)}\right)\right]-} \\
& {\left[\sum_{j=1}^{N} \sum_{\mathbf{s}} p_{\theta}\left(\mathbf{s} \mid \mathbf{o}^{(j)}\right) C_{k}\left(\mathbf{s}, \mathbf{o}^{(j)}\right)\right]-\frac{\lambda_{k}}{\sigma^{2}}, }
\end{aligned}
$$

where $C_{k}(\mathbf{s}, \mathbf{o})$ is the count of feature $f_{k}$ given $\mathbf{s}$ and $\mathbf{o}$, equal to $\sum_{t=1}^{T} f_{k}\left(s_{t-1}, s_{t}, \mathbf{o}, t\right)$, i.e. the sum of $f_{k}\left(s_{t-1}, s_{t}, \mathbf{o}, t\right)$ values for all positions, $t$, in the training sequence. The first two terms correspond to the difference between the empirical and the model expected values of feature $f_{k}$. The last term is the first-derivative of the Gaussian prior.

\section{IMPROVING DISCRIMINATIVE SEQUENTIAL LEARNING}

This section presents the proposed framework in detail: (1) how to discover rare-but-important associations from the training data and (2) how to integrate those associations

\begin{tabular}{|c|}
\hline \\
\hline $\begin{array}{l}\text { Sequential Training Data } \mathcal{D} \\
\left(\mathbf{o}^{(k-1)}, \mathbf{l}^{(k-1)}\right): \ldots \\
\left(\mathbf{o}^{(k)}, \mathbf{l}^{(k)}\right): \ldots \text { highly_RB ambiguous_JJ data_NNS ... } \\
\left(\mathbf{o}^{(k+1)}, \mathbf{l}^{(k+1)}\right): \ldots\end{array}$ \\
\hline 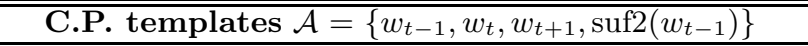 \\
\hline Transactional Database $\mathcal{T}_{D}$ \\
\hline $\begin{array}{l}\text { RB, } \ldots w_{t}: \text { highly, } w_{t+1} \text { :ambiguous, } \ldots \\
\text { JJ, } w_{t-1} \text { :highly, } w_{t} \text { :ambiguous, } w_{t+1} \text { :data, } \operatorname{suf} 2\left(w_{t-1}\right): \text { ly } \\
\text { NNS, } w_{t-1} \text { :ambiguous, } w_{t}: \text { data, } \ldots, \operatorname{suf} 2\left(w_{t-1}\right): \text { us } \\
\ldots\end{array}$ \\
\hline
\end{tabular}
into discriminative sequential learning models, e.g. CRFs.

\subsection{Mining Rare-but-Important Associations}

This section first presents the concept rare-but-important associations in discriminative sequential learning based on the traditional association rules [1], and then describes a method to discover such patterns from the training data.

\subsubsection{Associations in Sequential Training Data}

Table 1: Transactional database of POS tagging data

Recall that the training dataset for sequential learning is denoted as $\mathcal{D}=\left\{\left(\mathbf{o}^{(k)}, \mathbf{l}^{(k)}\right)\right\}_{k=1}^{N}$ where $\mathbf{o}^{(k)}$ and $\mathbf{l}^{(k)}$ are the $k^{\text {th }}$ data observation and label sequences, respectively. Let $\mathcal{A}=\left\{A_{1}, A_{2}, \ldots, A_{M}\right\}$ be the set of context predicate templates in which each template $A_{i}$ captures a particular type of contextual information about data observations. In 
a sense, $\mathcal{A}$ is similar to the set of attributes in a relational table. Applying all templates in $\mathcal{A}$ to each position in every training sequence $\left(\mathbf{o}^{(k)}, \mathbf{l}^{(k)}\right)$ in the training data $\mathcal{D}$, we obtain a transactional database $\mathcal{T}_{D}$ in which each transaction consists of a label and a list of active context predicates.

For example, the first part of Table 1 shows the training data $\mathcal{D}$ for POS tagging in which each training sequence $(\mathbf{o}, \mathbf{l})$ is an English sentence together with POS tags of words. The second part is a set of 4 context predicate templates: the identities of the previous word $\left(w_{t-1}\right)$, the current word $\left(w_{t}\right)$, the next word $\left(w_{t+1}\right)$, and the 2-character suffix of the previous word ( $\left.\operatorname{suf} 2\left(w_{i-1}\right)\right)$. The third part is the transactional database $\mathcal{T}_{D}$ after applying templates in $\mathcal{A}$ for $\mathcal{D}$.

Let $\mathcal{I}=\left\{x_{1}, x_{2}, \ldots, x_{n}\right\}$ be the set of all possible context predicates in the transactional database $\mathcal{T}_{D}$, let $\mathcal{L}$ be the set of all labels, and $\mathcal{T}=\left\{t_{1}, t_{2}, \ldots, t_{m}\right\}$ be the set of all transactions in $\mathcal{T}_{D}$. Our target is to examines every predictive association rule $r$ having the form below,

$$
X \Rightarrow l
$$

where the left hand side (LHS) of $r, X=\left\langle x_{i 1} \wedge x_{i 2} \wedge \ldots \wedge x_{i p}\right\rangle$ $\subset \mathcal{I}$, is a conjunction of $p$ context predicates in $\mathcal{I}$, and the right hand side (RHS) of rule $r$, i.e., $l \in \mathcal{L}$, is a particular label. The support of the rule $r$, denoted as $\sup (r)$, is the number of transactions in $\mathcal{T}$ containing $\{l\} \cup X$, and the confidence of $r$, denoted as $\operatorname{conf}(r)$, is the conditional probability that a transaction in $\mathcal{T}$ has the label $l$ given that it contains $X$, i.e., $\operatorname{conf}(r)=\sup (X \cup\{l\}) / \sup (X)$. In a sense, this kind of rule is similar to the associative classification rules in $[14,16]$ except that our work mainly focuses on rare-but-important associations as discussed in the next section.

\subsubsection{Rare-but-Important Associations}

Derived from the predictive association rules defined in (8) and the concepts of support and confidence factors, we present a descriptive definition of rare-but-confident associations below.

Definition 1. Let lsup and usup be two integers that are much smaller than the total number of transactions in $\mathcal{T}$ (i.e., lsup $\leq$ usup $\ll|\mathcal{T}|$ ), and let lconf be a real number that satisfies the condition $0 \leq \operatorname{lconf} \leq 1$ and lconf $\simeq 1$. A predictive association rule $r$ in (8) is called a rare-butconfident if:

$$
\operatorname{lsup} \leq \sup (r) \leq \operatorname{usup} \text { and } \operatorname{conf}(r) \geq \operatorname{lconf}
$$

All predictive association rules satisfying definition (1) are rare-but-confident. However, NOT all of them are important. This is based on the important observation that: "if most context predicates in the LHS of a rare-but-confident rule $r$ strongly support the label $l$, then the rule $r$ is trivial". In other words, if most context predicates in the LHS of $r$ largely support label $l$ in a separated manner, there is no need to examine the co-occurrence of all items in the LHS, and the model can still work properly without this rule. For example, in named entity recognition, the rule $\left\langle w_{t-1}\right.$ :New $\wedge w_{t}$ :York $\wedge w_{t+1}$ : weather $\Rightarrow$ label $_{t}=$ LOCATION $\rangle$ is not important because both " $w_{t-1}:$ New" and " $w_{t}$ :York" strongly support the label "LOCATION", and thus their conjunction should be unnecessary. In other words, the named entity recognizer can predict the label "LOCATION" for the word "York" without the above rule because both "New" and
"York" are frequently observed in the training data as a location name, i.e. "New York". Based on this observation, we define the concept of "rare-but-important" associations as follows,

Definition 2. A rare-but-confident rule $r: X \Rightarrow l$ is considered to be rare-but-important if there exists at least another label $l^{\prime} \in \mathcal{L}$ such that the sum of support counts for the label $l^{\prime}$ from the context predicates in the LHS of $r$ is larger than that for the label $l$, i.e.,

$$
\exists l^{\prime} \in \mathcal{L}: \sum_{x \in X} \sup \left(x \Rightarrow l^{\prime}\right)>\sum_{x \in X} \sup (x \Rightarrow l)
$$

Why are predictive association rules satisfying definition (2) important? Intuitively, if such a rule, $r$, exists in the training data but is not being discovered and emphasized, the model may predict the label $l^{\prime}$ for any data transaction holding all context predicates in the LHS of $r$ when the correct label is $l$. This is because most singleton context predicates in LHS of $r$ tend to support the label $l^{\prime}$ rather than $l$. This is why the appearance of predictive association rules satisfying definition (2) is important. There should be more sophisticated definitions and conditions of rare-butimportant predictive association rules. However, we choose the above definition because of the trade-off between the rigorousness and the simplicity of calculation.

For instance, the predictive association rule $\left\langle w_{t-1}: \mathrm{New}\right.$ $\wedge w_{t}$ :York $\wedge w_{t+1}$ :University $\Rightarrow$ label $_{t}=$ ORGANIZATION $\rangle$ is important for recognizing the named entity type of the current word ("York") since there is another label, "LOCATION", that should satisfy the condition addressed in definition (2), i.e., $\sum_{x \in X} \sup (x \Rightarrow$ LOCATION $)>\sum_{x \in X} \sup (x$ $\Rightarrow$ ORGANIZATION). This is because both New and York strongly support the label "LOCATION" rather than "ORGANIZATION". Thus, the appearance of the above rule can help the model to recognize "New York University" as an organization rather than a location.

\subsubsection{Discovering Rare-but-Important Associations}

Mining rare-but-important associations from the transactional database $\mathcal{T}_{D}$ encounters the following problems: (1) the number of data items, i.e., the number of atomic context predicates and labels $|\mathcal{I} \cup \mathcal{L}|$, is relatively large; and (2) the support thresholds, i.e., lsup and usup, are very small compared to the number of transactions $|\mathcal{T}|$. This means that there are a huge number of combinations of items that must be examined during the mining process.

Fortunately, FP-growth [11], a frequent pattern mining algorithm without candidate generation, can discover such associations in an acceptable computational time. This is because $\mathrm{FP}$-growth employs a $\mathrm{FP}$-tree (an extended prefix tree structure) to store crucial, quantitative information about frequent patterns in such a way that more frequently occurring items will have better chances of sharing nodes than less frequently occurring ones. All mining operations are then performed on the $\mathrm{FP}$-tree in a partitioning, recursive fashion without candidate generation. See [11] for a complete description of this algorithm.

Taking the sequential training data $\mathcal{D}=\left\{\left(\mathbf{o}^{(k)}, \mathbf{l}^{(k)}\right)\right\}_{k=1}^{N}$, the set of context predicate templates $\mathcal{A}=\left\{A_{1}, A_{2}, \ldots, A_{M}\right\}$, the lower and upper support thresholds lsup, usup (lsup $\leq$ usup $\ll|\mathcal{T}|)$, and the lower confidence threshold lconf $(0 \leq$ lconf $\leq 1$ and lconf $\simeq 1$ ) as inputs, Rare-but-important association mining includes the following steps: 
1. Transforming the sequential training data $\mathcal{D}$ to a transactional database $\mathcal{T}_{D}$ by applying all predicate templates in $\mathcal{A}$. $\mathcal{T}_{D}$ includes the set of items $\mathcal{I} \cup \mathcal{L}$ (all possible generated context predicates and all labels), the set of all transactions $\mathcal{T}$.

2. Mining all itemsets with supports larger or equal to lsup using the $\mathrm{FP}$-growth algorithm.

3. Generating all rare-but-confident association rules in the form of $X \Rightarrow l(8)$ with supports belonging to [lsup, usup], and the minimum confidence threshold lconf.

4. Selecting all possible rare-but-important association rules from rare-but-confident ones by applying the condition stated in definition (2).

In the fourth step, to determine whether or not a rarebut-confident rule, $r: X \Rightarrow l$, is rare-but-important, we have to scan the database to compute the sums of supports of context predicates in the LHS of $r$ for all other labels. This is an expensive operation. Fortunately, we can perform this on the $\mathrm{FP}$-tree by traversing the node-links of each label, starting at the header table, and looking upward and downward to count the supports from context predicates appearing in the LHS of $r$. See $[11,14]$ for the detailed description of FP-tree.

\subsection{Incorporating Rare-but-Important Asso- ciations into Conditional Random Fields}

This section presents three ways to incorporate the rarebut-important associations discovered from the training data into CRFs: (1) associations as normal features, (2) associations as features with emphasized feature functions, and (3) associations as constraints for the inference process.

\subsubsection{Rare-but-Important Associations as Normal Features of Conditional Random Fields}

All rare-but-important associations are in the form $X \Rightarrow$ $l$, in which $X=\left\langle x_{i 1} \wedge x_{i 2} \wedge \ldots \wedge x_{i p}\right\rangle(\subset \mathcal{I})$ is a conjunction of $p$ context predicates and $l \in \mathcal{L}$ is a particular label. These associations can be integrated into CRFs in terms of normal per-state features as follows.

$$
f_{k}^{(\text {per-state })}\left(s_{t}, \mathbf{o}, t\right)=\delta\left(s_{t}, l\right)\left\{x_{i 1}(\mathbf{o}, t) \wedge \ldots \wedge x_{i p}(\mathbf{o}, t)\right\}
$$

These per-state features are similar to those in (2) except that they capture a co-occurrence of $p$ atomic context predicates rather than a single one. The features are treated as normal features and are trained together.

\subsubsection{Rare-but-Important Associations as Normal Features with Weighted Feature Values}

It is noticeable that rare-but-important features are infrequently observed in the training data, and thus their learned weights should be small. This means that their contributions, in several cases, may not be sufficient to win the dominance of common statistics, i.e., frequently observed singleton features. To overcome this drawback, we emphasize rare-but-important features by assigning larger feature function values compared to normal features.

$$
f_{k}^{(\text {per-state })}\left(s_{t}, \mathbf{o}, t\right)= \begin{cases}v & \text { if } \delta\left(s_{t}, l\right) \text { and } \\ & \left\{x_{i 1}(\mathbf{o}, t) \wedge \ldots \wedge x_{i p}(\mathbf{o}, t)\right\} \\ 0 & \text { otherwise }\end{cases}
$$

where $\delta\left(s_{t}, l\right)$ and $\left\{x_{i 1}(\mathbf{o}, t) \wedge \ldots \wedge x_{i p}(\mathbf{o}, t)\right\}$ are considered as logic expressions, and $v$ is larger than 1 (the feature value of normal features). $v$ should be large if the occurrence frequency of the feature (also the support of the rare-butimportant association) is small. Thus, for each feature generated from a rare-but-important association $r, v$ is equal to $(\operatorname{usup}-\sup (r)+2)$. This ensures that $v$ is always bigger than 1 and inversely proportional to the support of $r$, i.e., the occurrence frequency of the feature.

\subsubsection{Rare-but-Important Associations as Constraints for the Inference Process}

Constrained CRFs are extensions of CRFs in which useful constraints are incorporated into the inference process (i.e., the Viterbi algorithm) to correct potential errors existing in the most likely output state sequence for each input observation sequence. Kristjansson et al. [8] proposed this extension with the application to interactive form filling, in which users can examine the filling process and make necessary corrections in terms of their own constraints. A re-correction applied at a particular position will propagate though the Viterbi sequence to make automatic updates for labels at other positions, i.e., the correction propagation capability.

This section presents the integration of rare-but-important associations with 100\%-confidence into the Viterbi algorithm in terms of data-driven constraints to make corrections directly to the inference process of CRFs. Unlike those used in [8], our constraints are $100 \%$-confidence associations and are automatically discovered from the training data.

Normally, CRFs use a variant of the traditional Viterbi algorithm to find the most likely state sequence given an input observation sequence. To avoid an exponential-time search over all possible settings of state sequence, this algorithm employs a dynamic programming technique with a forward variable $\varphi_{t+1}\left(s_{i}\right)$ in definition (5).

Let $R=\left\{r_{1}, r_{2}, \ldots, r_{q}\right\}$ be a set of $q$ rare-but-important associations with 100\%-confidence, and each $r_{u}(1 \leq u \leq q)$ has the form $\left\langle x_{u 1} \wedge x_{u 2} \wedge \ldots \wedge x_{u p}\right\rangle \Rightarrow l_{u}\left(l_{u} \in \overline{\mathcal{L}}\right)$. Each $r_{u} \in R$ is considered to be a constraint for the inference process. At each time position in the testing data sequence, we check whether or not the set of active context predicates at the current position holds the LHS of any rule $r_{u} \in R$. If yes, the most likely state path must go though the current state with the label $l_{u}$ (i.e., the RHS or rule $r_{u}$ ), and the possibility of passing though other labels equals to zero. The constrained forward variable is re-defined as follows.

$$
\varphi_{t+1}\left(s_{i}\right)=\left\{\begin{array}{l}
\max _{s_{j}}\left\{\varphi_{t}\left(s_{j}\right) \exp \left[\sum_{k} \lambda_{k} f_{k}\left(s_{j}, s_{i}, \mathbf{o}, t\right)\right]\right\} \\
\text { if } \delta\left(s_{i}, l_{u}\right) \text { and }\left\{x_{u 1}(\mathbf{o}, t) \wedge \ldots \wedge x_{u p}(\mathbf{o}, t)\right\} \\
0 \text { otherwise }
\end{array}\right.
$$

The constraint applied at the time position $t$ will propagate though the whole sequence and make some re-corrections for labels at other positions (mostly around the position $t$ ).

One problem is that when the number of constraints (i.e., the number of $100 \%$-confidence rare-but-important predictive association rules) is large, the time for examining the LHS of every rule at each position in the testing sequence also becomes large. To overcome this obstacle, we propose the following algorithm for a fast checking for constraints at a particular time position $t$ in the testing sequence. 
Let $R=\left\{r_{1}, r_{2}, \ldots, r_{q}\right\}$ be the set of $100 \%$-confidence rules, also known as constraints, and let $X=\left\{x_{1}, x_{2}, \ldots, x_{m}\right\}$ be the set of $m$ active context predicates observed at the current position $t$. The target of the following algorithm is to check whether or not $X$ holds the LHS of any constraint $r_{u}$ $\in R$. If yes, choose the constraint with the longest LHS.

1. For each $x_{i} \in X$, lookup the set of constraints $R_{i} \subset R$ in which the LHS of every constraint in $R_{i}$ contains $x_{i}$. Denote $R^{\prime}=\left\{R_{1} \cup R_{2} \cup \ldots \cup R_{m}\right\}$.

2. For each constraint $r_{j} \in R^{\prime}$, let $c_{j}$ be the sum of occurrence frequency of $r_{j}$ in $R_{1}, R_{2}, \ldots, R_{m}$.

3. Find the pair $\left\langle r_{j}, c_{j}\right\rangle\left(1 \leq j \leq\left|R^{\prime}\right|\right)$ such that $c_{j}$ is the largest number satisfying the condition: $c_{j}$ equals to the number of all context predicates in the LHS of $r_{j}$.

If this algorithm find a constraint $r_{j}$, then apply this constraint to the current position $t$ with formula (9), otherwise, apply the normal Viterbi recursion as formula (5).

\section{EXPERIMENTAL RESULTS}

\subsection{Experimental Settings}

All the experiments were performed with our $\mathrm{C} / \mathrm{C}++$ implementation of CRFs - FlexCRFs ${ }^{2}$ - on $2.5 \mathrm{GHz}, 1 \mathrm{~Gb}$ RAM, Pentium IV processor with RedHat Linux. All CRF models were trained using the limited-memory quasi-Newton method for unconstrained optimization, L-BFGS [15]. Unlike those used in [25], our CRF models are simpler and easier to implement by obeying the first-order Markov property, i.e., the label of the current state depends only on the label of the previous state.

Training and testing data for English phrase chunking and named entity recognition can be found at the shared tasks of CoNLL2000 ${ }^{3}$ and CoNLL2003 ${ }^{4}$, respectively.

\subsection{Phrase Segmentation}

Phrase chunking, an intermediate step toward full parsing of natural language, identifies phrase types (e.g., noun phrase - NP, verb phrase - VP, $\mathrm{PP}$ - prepositional phrase, etc.) in text sentences. Here is an example of a sentence with phrase marking: "[NP He] [VP reckons] [NP the current account deficit] [VP will narrow] [PP to] [NP only \# 1.8 billion] [PP in] [NP September]."

\subsubsection{Training and Testing Data}

The training and testing data for this task is available at the shared task for CoNLL-2000. The data consist of the same partitions of the Wall Street Journal corpus (WSJ): sections $15-18$ as training data (8936 sentences, 211727 tokens) and section 20 as testing data (2012 sentences, 47377 tokens). Each line in the annotated data is for a token and consists of three columns: the token (a word or a punctuation mark), the part-of-speech tag of the token, and the phrase type label (label for short) of the token. The label of each token indicates whether the token is outside a phrase $(\mathrm{O})$, starts a phrase (B-〈PhraseType $\rangle)$, or continues

\footnotetext{
${ }^{2}$ The documents and source code of FlexCRFs are available at http://www.jaist.ac.jp/ hieuxuan/flexcrfs/flexcrfs.html ${ }^{3}$ http://cnts.uia.ac.be/conll2000/chunking/

${ }^{4}$ http://cnts.uia.ac.be/conll2003/ner/
}

a phrase (I-〈PhraseType $\rangle)$. For example, the label sequence of the above sentence is " $\mathrm{B}-\mathrm{NP} \mathrm{B}-\mathrm{VP} \mathrm{B}-\mathrm{NP} \mathrm{I}-\mathrm{NP} \mathrm{I}-\mathrm{NP} \mathrm{I}-$ NP B-VP I-VP B-PP B-NP I-NP I-NP I-NP B-PP B-NP O". This dataset contains 11 phrase types as shown in the first column of Table 3. Two consecutive data sequences (sentences) are separated by a blank line.

\subsubsection{Feature Selection}

On the phrase chunking dataset, we use feature templates as shown in Table 2. All transition features obey the firstorder Markov dependency that the label $(l)$ of the current state depends on the label $\left(l^{\prime}\right)$ of the previous state (e.g., $" l=\mathrm{I}-\mathrm{NP} "$ and " $l$ ' $=\mathrm{B}-\mathrm{NP} ")$. Each per-state feature expresses how much influence a context predicate $(x(\mathbf{o}, t))$ observed surrounding the current position $t$ has on the label (l) of the current state. A context predicate captures a particular property of the observation sequence. For instance, the per-state feature " $l=\mathrm{I}-\mathrm{NP}$ " and " $\operatorname{word}_{t-1}$ is the" indicates that the label of the current state should be I-NP (i.e., continue a noun phrase) if the previous word is the.

Table 3 describes both transition and per-state feature templates. Context predicates for per-state features are identities of words, POS tags of words surrounding the current position $t$, such as words and POS tags at positions $t-2, t-1, t, t+1, t+2$ (i.e., window size is 5 ).

Table 2: Feature templates for phrase chunking

\begin{tabular}{|c|c|}
\hline \multicolumn{2}{|c|}{ Transition feature templates } \\
\hline Current state: $s_{t}$ & Previous state: $s_{t-1}$ \\
\hline$l$ & $l^{\prime}$ \\
\hline \hline \multicolumn{2}{|c|}{ Per-state feature templates } \\
\hline Current state: $s_{t}$ & Context predicate: $x(\mathbf{o}, t)$ \\
\hline$l$ & $w_{t-2} ; w_{t-1} ; w_{t} ; w_{t+1} ; w_{t+2}$ \\
& $w_{t-1} \wedge w_{t} ; w_{t} \wedge w_{t+1}$ \\
& $p_{t-2} ; p_{t-1} ; p_{t} ; p_{t+1} ; p_{t+2}$ \\
& $p_{t-2} \wedge p_{t-1} ; p_{t-1} \wedge p_{t}$ \\
& $p_{t} \wedge p_{t+1} ; p_{t+1} \wedge p_{t+2}$ \\
& $p_{t-2} \wedge p_{t-1} \wedge p_{t}$ \\
& $p_{t-1} \wedge p_{t} \wedge p_{t+1}$ \\
& $p_{t} \wedge p_{t+1} \wedge p_{t+2}$ \\
\hline
\end{tabular}

We also employ 2-order conjunctions of the current word with the previous $\left(w_{t-1} \wedge w_{t}\right)$ or the next word $\left(w_{t} \wedge w_{t+1}\right)$, and 2-order and 3-order conjunctions of two or three consecutive POS tags within the current window to make use of the mutual dependencies among singleton properties.

With the feature templates shown in Table 2 and the feature rare threshold of 1 (i.e., only features with occurrence frequency larger than 1 are included into the CRF model), 321526 context predicates and $152856 \mathrm{CRF}$ features were generated from 8936 training data sequences.

\subsubsection{Mining Rare-but-Important Associations}

Let $\mathcal{I}$ be the itemset of 321548 data items, i.e., the union set of 321526 context predicates and 22 phrase labels; $\mathcal{T}$ be the set of 211727 data transactions corresponding to 211727 tokens of the training data (the maximum transaction length is 20 , i.e., 19 context predicate templates plus the label). Let the lower support (lsup) and upper support (usup) thresholds be 4 and 8 , respectively; the lower confidence (lconf) threshold be 0.98 or $98 \%$. In fact, all output rules have the confidence of $100 \%$ because lconf $=0.98>\frac{7}{8}$ and there- 
fore larger than all other confidence levels. We also confine the length of the LHS of all rare-but-important associations between 3 and 6 . There are several reasons why we confine the LHS length between 3 and 6 . First, although simple rules (i.e., with shorter LHS length) are usually useful for generalization, we only examine rules complex enough (with LHS length $\geq 3$ ) because our main target is to deal with hard data instances which are not frequently observed in the training data. We also observed that the number of rare-but-important with LHS length smaller than 3 was small. Second, rules with LHS length larger than 6 are usually too specific and most of them are covered by rules with LHS length of 4,5 or 6 . Also, mining and generating all long rules is time-consuming. For these reasons, we only considered rules with LHS length between 3 and 6 .

The mining process for rare-but-important associations took 2 hours using FP-growth algorithm and the filter criteria presented in definitions (1) and (2). The output was a set of 10364 rare-but-important associations with an LHS length between 3 and 6 , support between 4 and 8 , and confidence of $100 \%$. This set of associations were integrated into the CRF model in terms of normal features, normal features with weighted feature values, and constraints for the inference process.

\subsubsection{Results}

Table 3: The performance of English phrase chunking without rare-but-important associations

\begin{tabular}{|c|r|r|r|r|r|r|}
\hline Phrase & \#Hm. & \#Ml. & \#Mt. & Pr. & Rc. & F1. \\
\hline NP & 12422 & 12399 & 11613 & 93.66 & 93.49 & 93.57 \\
PP & 4811 & 4832 & 4684 & 96.94 & 97.36 & 97.15 \\
VP & 4658 & 4690 & 4375 & 93.28 & 93.92 & 93.60 \\
SBAR & 535 & 538 & 459 & 85.32 & 85.79 & 85.55 \\
ADJP & 438 & 398 & 303 & 76.13 & 69.18 & 72.49 \\
ADVP & 866 & 864 & 686 & 79.40 & 79.21 & 79.31 \\
PRT & 106 & 95 & 75 & 78.95 & 70.75 & 74.63 \\
LST & 5 & 0 & 0 & 0.00 & 0.00 & 0.00 \\
INTJ & 2 & 1 & 1 & 100.0 & 50.00 & 66.67 \\
CONJP & 9 & 16 & 6 & 37.50 & 66.67 & 48.00 \\
UCP & 0 & 0 & 0 & 0.00 & 0.00 & 0.00 \\
\hline Avg1. & & & & 74.12 & 70.64 & 72.34 \\
Avg2. & 23852 & 23833 & 22202 & 93.16 & 93.08 & 93.12 \\
\hline
\end{tabular}

Table 4: The performance of English phrase chunking with rare-but-important associations as normal features of CRFs

\begin{tabular}{|c|r|r|r|r|r|r|}
\hline Phrase & \#Hm. & \#Ml. & \#Mt. & Pr. & Rc. & F1. \\
\hline NP & 12422 & 12402 & 11645 & 93.90 & 93.74 & 93.82 \\
PP & 4811 & 4849 & 4686 & 96.64 & 97.40 & 97.02 \\
VP & 4658 & 4687 & 4381 & 93.47 & 94.05 & 93.76 \\
SBAR & 535 & 522 & 473 & 90.61 & 88.41 & 89.50 \\
ADJP & 438 & 416 & 337 & 81.01 & 76.94 & 78.92 \\
ADVP & 866 & 850 & 726 & 85.41 & 83.83 & 84.62 \\
PRT & 106 & 104 & 76 & 73.08 & 71.70 & 72.38 \\
LST & 5 & 3 & 1 & 33.33 & 20.00 & 25.00 \\
INTJ & 2 & 1 & 1 & 100.0 & 50.00 & 66.67 \\
CONJP & 9 & 11 & 7 & 63.64 & 77.78 & 70.00 \\
UCP & 0 & 0 & 0 & 0.00 & 0.00 & 0.00 \\
\hline Avg1. & & & & 81.11 & 75.39 & 78.14 \\
Agv2. & 23852 & 23845 & 22333 & 93.66 & 93.63 & 93.65 \\
\hline
\end{tabular}

Table 5: The performance of English phrase chunking with rare-but-important associations as normal features with weighted feature values

\begin{tabular}{|c|r|r|r|r|r|r|}
\hline Phrase & \#Hm. & \#Ml. & \#Mt. & Pr. & Rc. & F1. \\
\hline NP & 12422 & 12398 & 11659 & 94.04 & 93.86 & 93.95 \\
PP & 4811 & 4851 & 4694 & 96.76 & 97.57 & 97.16 \\
VP & 4658 & 4683 & 4385 & 93.64 & 94.14 & 93.89 \\
SBAR & 535 & 524 & 473 & 90.27 & 88.41 & 89.33 \\
ADJP & 438 & 415 & 339 & 81.69 & 77.40 & 79.48 \\
ADVP & 866 & 853 & 726 & 85.11 & 83.83 & 84.47 \\
PRT & 106 & 103 & 79 & 76.70 & 74.53 & 75.60 \\
LST & 5 & 4 & 3 & 75.00 & 60.00 & 66.67 \\
INTJ & 2 & 1 & 1 & 100.0 & 50.00 & 66.67 \\
CONJP & 9 & 10 & 6 & 60.00 & 66.67 & 63.16 \\
UCP & 0 & 0 & 0 & 0.00 & 0.00 & 0.00 \\
\hline Avg1. & & & & 85.32 & 78.64 & 81.84 \\
Agv2. & 23852 & 23842 & 22365 & 93.81 & 93.77 & 93.79 \\
\hline
\end{tabular}

Table 6: The performance of English phrase chunking with rare-but-important associations as constraints for inference

\begin{tabular}{|c|r|r|r|r|r|r|}
\hline Phrase & \#Hm. & \#Ml. & \#Mt. & Pr. & Rc. & F1. \\
\hline NP & 12422 & 12401 & 11662 & 94.04 & 93.88 & 93.96 \\
PP & 4811 & 4852 & 4697 & 96.81 & 97.63 & 97.22 \\
VP & 4658 & 4683 & 4385 & 93.64 & 94.14 & 93.89 \\
SBAR & 535 & 524 & 475 & 90.65 & 88.79 & 89.71 \\
ADJP & 438 & 419 & 342 & 81.62 & 78.08 & 79.81 \\
ADVP & 866 & 853 & 724 & 84.88 & 83.60 & 84.24 \\
PRT & 106 & 103 & 81 & 78.64 & 76.42 & 77.51 \\
LST & 5 & 4 & 3 & 75.00 & 60.00 & 66.67 \\
INTJ & 2 & 2 & 1 & 50.00 & 50.00 & 50.00 \\
CONJP & 9 & 9 & 7 & 77.78 & 77.78 & 77.78 \\
UCP & 0 & 0 & 0 & 0.00 & 0.00 & 0.00 \\
\hline Avg1. & & & & 82.31 & 80.03 & 81.15 \\
Agv2. & 23852 & 23850 & 22377 & 93.82 & 93.82 & 93.82 \\
\hline
\end{tabular}

Table 3 shows the highest performance (achieved at the $48^{\text {th }}$ L-BFGS iteration) of the phrase chunking task trained on the original set of $152856 \mathrm{CRF}$ features without rarebut-important associations. In each line, the first column is the phrase type; the second (\#Hm.) is the number of human annotated phrases; the third (\#Ml.) is the number of phrases automatically marked by the CRF model; the fourth (\#Mt.) is the number of correct phrases marked by the model; the last three columns are precision (Pr.), recall (Rc.), and F1-measure (F1), respectively. The last two lines are the average performance calculated in two ways: precision-recall based and phrase based; the first is based on the precision and recall values of separated phrase types and the second is based on the average numbers of humanannotated, model, and correct phrases. The first average F1 $(72.34 \%)$ reflects the balance and the trade-off among perlabel performances while the second average F1 (93.12\%) reflects the total performance.

Table 4, which has the same format as Table 3, describes the performance of phrase segmentation when discovered rare-but-important associations were integrated into the CRF model as normal features. The highest average F1measure achieved at the $45^{t h}$ L-BFGS iteration is $93.65 \%$, i.e., $0.53 \%$ higher than the original performance. 
Table 5 shows the performance when all rare-but-important associations were incorporated into the CRF model in the form of features with weighted feature values. The highest average $\mathrm{F} 1-$ measure (at the $47^{\text {th }}$ iteration) is $93.79 \%$, i.e., $0.67 \%$ higher than the original performance.

Table 6 describes the performance when all 100\%-confidence rare-but-important associations were used as constraints for the inference process. The highest average F1-measure is $93.82 \%$, i.e., $0.70 \%$ higher than the original performance.

\subsection{Named Entity Recognition}

Named entity recognition (NER), a subtask of information extraction, identifies names of persons (PER), organizations (ORG), locations (LOC), times (DATE/TIME), and quantities (NUMBER, CURRENCY, PERCENTAGE) in natural language. Here is an example of an English sentence with named entities marked: "[LOC Germany] 's representative to the [ORG European Union] 's veterinary committee [PER Werner Zwingmann] said on Wednesday ..."

\subsubsection{Training and Testing Data}

The training and testing data for English named entity recognition are provided at the shared task for CoNLL-2003. The dataset is a collection of news wire articles from the Reuters Corpus. The training set consists of 14041 sentences (203621 tokens), and the testing data contains two parts: the development test set (testa: 3250 sentences, 51362 tokens) and the final test set (testb: 3453 sentences, 46435 tokens). The data files contain four columns separated by a blank space. Each token (a word or a punctuation mark) has been put on a separate line and there is an empty line after each sentence (sequence). The first item on each line is a token, the second is the part-of-speech tag of the token, the third is a phrase type tag (like the label in phrase chunking) of the token, and the fourth is the named entity label (label for short). The label of each token indicates whether the token is outside a named entity $(\mathrm{O})$, or inside a named entity (I-〈NamedEntityType $\rangle$ ). If two named entities of the same type immediately follow each other, the first token of the second named entity will have tag B-〈NamedEntityType〉. For example, the named entity label sequence of the above sentence is "I-LOC O O O O I-ORG I-ORG O O O I-PER I-PER O O O ...".

\subsubsection{Feature Selection}

On the named entity recognition dataset, we used the feature templates shown in Table 7. All transition features also conform to the first Markovian property. Each context predicate for a per-state feature is one of the following types: (1) the identities of words $\left(w_{t-2}, w_{t-1}, w_{t}, w_{t+1}, w_{t+2}\right),(2)$ the POS tags of words $\left(p_{t-2}, p_{t-1}, p_{t}, p_{t+1}, p_{t+2}\right),(3)$ the phrase tags of words $\left(c_{t-2}, c_{t-1}, c_{t}, c_{t+1}, c_{t+2}\right)$, and (4) several simple regular expressions or formats of words such as "the first character of a word is capitalized" (IsInitialCapitalized), "all chars of a word are capitalized" (IsAllCapitalized), etc. Like the phrase chunking task, all context predicates are captured within a window with size of 5 . Our feature templates are simpler than those used in the previous work presented at the CoNLL2003 shared task and in [19] in two ways: only five simple format properties were captured (compared to 16 regular expressions in [19]), and no external dictionaries were used such as the lists of people names, organization names, countries, cities, etc.
With the feature templates described in Table 7 and the feature rare threshold of 1,125206 context predicates and 77826 features were generated from 14041 training sequences.

Table 7: Feature templates for NER

\begin{tabular}{|c|c|}
\hline \multicolumn{2}{|c|}{ Transition feature templates } \\
\hline Current state: $s_{t}$ & Previous state: $s_{t-1}$ \\
\hline$l$ & $l^{\prime}$ \\
\hline \hline \multicolumn{2}{|c|}{ Per-state feature templates } \\
\hline Current state: $s_{t}$ & Context predicate: $x(\mathbf{o}, t)$ \\
\hline$l$ & $w_{t-2} ; w_{t-1} ; w_{t} ; w_{t+1} ; w_{t+2}$ \\
& $p_{t-2} ; p_{t-1} ; p_{t} ; p_{t+1} ; p_{t+2}$ \\
& $c_{t-2} ; c_{t-1} ; c_{t} ; c_{t+1} ; c_{t+2}$ \\
& IsInitialCapitalized $\left(w_{k}\right)$ \\
& IsAllCapitalized $\left(w_{k}\right)$ \\
& IsNumber $\left(w_{k}\right)$ \\
& IsAlphaNumber $\left(w_{k}\right)$ \\
& IsFirstWord $\left(w_{k}\right)$ \\
& where $k \in\{t-2, t-1, t, t+1, t+2\}$ \\
\hline
\end{tabular}

\subsubsection{Mining Rare-but-Important Associations}

Let $\mathcal{I}$ be the itemset of 125215 data items, i.e., the union set of 125206 context predicates and 9 named entity labels; $\mathcal{T}$ be the set of 203621 data transactions corresponding to 203621 tokens of the training data (the maximum transaction length is 41 , i.e., 40 context predicate templates plus the label). Let the lower support (lsup) and upper support (usup) thresholds be 4 and 8 , respectively; the lower confidence (lconf) threshold be 0.98 or $98 \%$. We also examine rules with the LHS length between 3 and 6 .

The mining process for rare-but-important associations took about 1.5 hours using $\mathrm{FP}$-growth algorithm and the filter criteria described in definitions (1) and (2). The output was a set of 9023 rare-but-important associations with an LHS length between 3 and 6 , support between 4 and 8 , and confidence of $100 \%$. This set of associations was integrated into the CRF model in terms of normal features, normal features with weighted feature values, and constraints for the inference process.

\subsubsection{Results}

Table 8: The performance of English named entity recognition without rare-but-important associations

\begin{tabular}{|c|r|r|r|r|r|r|}
\hline NEType & \#Hm. & \#Ml. & \#Mt. & Pr. & Rc. & F1. \\
\hline ORG & 1325 & 1254 & 1043 & 83.17 & 78.72 & 80.88 \\
PER & 1829 & 1806 & 1616 & 89.48 & 88.35 & 88.91 \\
LOC & 1832 & 1829 & 1636 & 89.45 & 89.30 & 89.37 \\
MISC & 916 & 852 & 735 & 86.27 & 80.24 & 83.14 \\
\hline Avg1. & & & & 87.09 & 84.15 & 85.60 \\
Avg2. & 5902 & 5741 & 5030 & 87.62 & 85.23 & 86.40 \\
\hline
\end{tabular}

Table 8 shows the highest performance (F1 of $86.40 \%$, achieved at the $133^{\text {th }}$ L-BFGS iteration) of the NER task trained on the original set of $77826 \mathrm{CRF}$ features. This table has the same format as Table 3 except that the first column of each line is the named entity type.

Table 9, which has the same format as Table 8, displays the experimental results of NER when all rare-but-important 
Table 9: The performance of English named entity recognition with rare-but-important associations as normal features of CRFs

\begin{tabular}{|c|r|r|r|r|r|r|}
\hline NEType & \#Hm. & \#Ml. & \#Mt. & Pr. & Rc. & F1. \\
\hline ORG & 1325 & 1256 & 1104 & 87.90 & 83.32 & 85.55 \\
PER & 1829 & 1811 & 1628 & 89.90 & 89.01 & 89.45 \\
LOC & 1832 & 1825 & 1647 & 90.25 & 89.90 & 90.07 \\
MISC & 916 & 855 & 757 & 88.54 & 82.64 & 85.49 \\
\hline Avg1. & & & & 89.14 & 86.22 & 87.66 \\
Agv2. & 5902 & 5747 & 5136 & 89.37 & 87.02 & 88.18 \\
\hline
\end{tabular}

Table 10: The performance of English named entity recognition with rare-but-important associations as normal features with weighted feature values

\begin{tabular}{|c|r|r|r|r|r|r|}
\hline NEType & \#Hm. & \#Ml. & \#Mt. & Pr. & Rc. & F1. \\
\hline ORG & 1325 & 1250 & 1108 & 88.64 & 83.62 & 86.06 \\
PER & 1829 & 1809 & 1633 & 90.27 & 89.28 & 89.77 \\
LOC & 1832 & 1827 & 1652 & 90.42 & 90.17 & 90.30 \\
MISC & 916 & 853 & 757 & 88.75 & 82.64 & 85.59 \\
\hline Avg1. & & & & 89.52 & 86.43 & 87.95 \\
Agv2. & 5902 & 5739 & 5150 & 89.74 & 87.26 & 88.48 \\
\hline
\end{tabular}

associations were integrated into $\mathrm{CRF}$ model in terms of normal features. The highest $\mathrm{F} 1-$ measure is $88.18 \%$, i.e., $1.78 \%$ higher than the original performance. Table 10 shows the results of NER in the case rare-but-important associations were encoded into the model in the form of normal features with weighted values. The highest average F1 is $88.48 \%$. Table 11 demonstrates the performance when all $100 \%$-confidence rare-but-important associations were integrated into the inference process in terms of Viterbi constraints. The highest F1 obtained in this case is $88.51 \%$.

\subsection{Discussion}

We can see that the integration of rare-but-important associations into CRF models can improve the performance of both the phrase chunking and named entity recognition tasks. The F1-measure (Avg2.) of phrase chunking increases from $93.12 \%$ to $93.65 \%, 93.79 \%$, and $93.82 \%$ corresponding to three methods of encoding rare-but-important associations. Similarly, the F1-measure of NER increases from $86.40 \%$ to $88.18 \%, 88.48 \%$, and $88.51 \%$. The precisionrecall based F1-measure (Avg1.) also increases from $72.34 \%$ to $78.14 \%, 81.84 \%$, and $81.15 \%$ for phrase chunking and from $85.60 \%$ to $87.66 \%, 87.95 \%$, and $87.99 \%$ for named entity recognition. This demonstrates that our approach can improve not only total performance but also the balance among classes/labels.

We can also draw some conclusions from the experimental results: (1) rare-but-important associations as normal CRF features (the first method) can significantly enhance the total performance; however, treating rare-but-important associations as normal features can not fully utilizes their advantages; (2) rare-but-important associations as constraints for inference (the third method) are sometimes too aggressive because they are globally true on one training dataset but may not be true on another; and (3) treating rarebut-important associations as normal features with emphasized values should be the favorable choice because they are neither too loosely nor too tightly integrated with the
Table 11: The performance of English named entity recognition with rare-but-important associations as constraints for inference

\begin{tabular}{|c|r|r|r|r|r|r|}
\hline NEType & \#Hm. & \#Ml. & \#Mt. & Pr. & Rc. & F1. \\
\hline ORG & 1325 & 1255 & 1112 & 88.61 & 83.92 & 86.20 \\
PER & 1829 & 1807 & 1630 & 90.20 & 89.12 & 89.66 \\
LOC & 1832 & 1829 & 1655 & 90.49 & 90.34 & 90.41 \\
MISC & 916 & 855 & 758 & 88.65 & 82.75 & 85.60 \\
\hline Avg1. & & & & 89.49 & 86.53 & 87.99 \\
Agv2. & 5902 & 5746 & 5155 & 89.71 & 87.34 & 88.51 \\
\hline
\end{tabular}

models. The experimental results show that this method achieves both high total performance and a balance among classes/labels.

We also did the experiments with rare-but-confident rules. We observed two important points that (1) the numbers of rare-but-important rules (both noun phrase chunking and named entity recogniztion) were much larger than those of rare-but-important ones; and (2) the experimental results were sometimes worse because of the overfitting problem. This means that there is a large proportion of rare-butimportant rules that are unnecessary for capturing difficult data instances.

The experimental results reported in this paper do not represent the best possible performances on phrase chunking and named entity recognition because: (1) our feature templates are relatively simple to keep the set of features compact; this is convenient for mining associations, training again and again during conducting the experiments; (2) unlike the CRF model in [25], all our CRF models obey the first-order Markov property to reduce the number of features and the training time.

\section{RELATED WORK}

Discriminative (sequential) learning models have been applied successfully in different natural language processing and information extraction tasks, such as POS tagging [24], text chunking [20, 25], information extraction [8, 22], computer vision and image analysis $[10,12,26]$, and biological modeling [27]. Normally, one can extract features from sequential data within a relatively large window size (i.e., the history size of contextual information) and make high-order combinations of atomic observational tests (e.g., the conjunctions of two or three consecutive words in a sentence) in the hope that they will capture as many useful predictive clues as possible. Unfortunately, such useful conjunctions are sparsely distributed in the feature space, and thus one unintentionally includes a large number of redundant conjunctions into the model. Inspired by this obstacle, our work aims at picking up useful conjunctions from a large array of conjunction candidates while keeping the set of features simple. The data-driven search with respect to support and confidence factors based on association rule mining techniques can discover desired conjunctions with an acceptable computational time.

McCallum [19] proposed an automated feature induction for CRFs that can dramatically reduce the number of used features. This likelihood-driven approach repeatedly adds features with high likelihood gains into the model. The set of induced features contains both atomic observational tests and conjunctions of them. The main difference between this 
work and ours is that McCallum focuses on features with high likelihood-gains in order to reduce the number of used features as much as possible, while the main target of our method is to discover rare-but-important associations or co-occurrences of weak statistics from the training data to highlight difficult examples. Further, our method can examine any combination or conjunction of context predicates because of the exhaustive working method of association rule mining techniques.

An error-driven method that combines boosting technique into the training process of CRFs [2] to minimize an upper bound on the ranking loss that was adapted to label sequences. This method also focuses on hard observation sequences, but without integrating new useful conjunctions of basic features. Another boosting-like training for CRFs is based on the use of "gradient tree" [6] to learn many conjunctions of features. One problem is that this method requires adding many trees for the training process.

\section{CONCLUSIONS AND FUTURE WORK}

In this paper, we proposed a data-driven approach that can discover and highlight rare-but-important associations or co-occurrences of singleton context predicates from the sequential training data to deal with hard examples. Discovered associations are integrated into the exponentiallytrained sequential learning models as normal features, features with weighted values, and constraints for the inference process. The experimental results show that rare-butimportant associations can improve the model performance by fighting against the dominance of singleton but common statistics in the training data.

Though rare-but-important associations can enhance the prediction accuracy for hard examples, our approach is currently based on the occurrence frequency of statistics and the existence of rare-but-important associations in the training data. We believe that there is an indirect theoretical relation between the occurrence frequencies of statistics and the learned weights of the model's features. Our future work will focus on this potential relation to estimate the extent to which useful patterns (e.g., rare-but-important associations) discovered from the training data can improve the performance of discriminative (sequential) learning models.

\section{ACKNOWLEDGMENTS}

We would like to thank Dr. Bart Goethals, Department of Math and Computer Science, Antwerpen University, for sharing his lightweight and efficient implementation of the FP-growth algorithm. We would like to say thank you to Prof. Jorge Nocedal, Department of Electrical and Computer Engineering, School of Engineering and Applied Science, Northwestern University, the author of FORTRAN implementation of the L-BFGS optimization procedure. We also would like to thank Prof. Sunita Sarawagi, KR School of Information Technology, IIT Bombay, the author of the Java CRFs package, which is the precursor of our $\mathrm{C} / \mathrm{C}++$ CRFs toolkit.

\section{REFERENCES}

[1] R. Agrawal and R. Srikant. Fast algorithms for mining association rules. In Proc. VLDB, pages 487-499, 1994.

[2] Y. Altun, T. Hofmann, and M. Johnson. Discriminative learning for label sequences via boosting. In Proc. NIPS, 2002.
[3] A. Berger, A.D. Pietra, and J.D. Pietra. A maximum entropy approach to natural langauge processing. Computational Linguistics, 22(1):39-71, 1996.

[4] S.F. Chen and R. Rosenfeld. A gaussian prior for smoothing maximum entropy models. Technical Report CMU-CS-99-108, CMU, 1999.

[5] M. Collins. Discriminative training methods for hidden markov models: theory and experiment with perceptron algorithms. In Proc. EMNLP, 2002.

[6] T.G. Dietterich. Training conditional random fields via gradient tree boosting. In Proc. ICML, 2004.

[7] Y. Freund and R. Schapire. A decision-theoretic generalization of on-line learning and application to boosting. Journal of Computer and System Sciences, 55:119-139, 1997.

[8] T. Kristjansson, A. Culotta, P. Viola, and A. McCallum. Interactive information extraction with constrained conditional random fields. In Proc. AAAI, pages 412-418, 2004.

[9] T. Kudo and Y. Matsumoto. Chunking with support vector machines. In Proc. ACL/NAACL, 2001.

[10] S. Kumar and M. Hebert. Discriminative random fields: a discriminative framework for contextual interaction in classification. In Proc. IEEE CVPR, pages 1150-1157, 2003.

[11] J. Han, J. Pei, and Y. Yin. Mining frequent patterns without candidate generation. In Proc. ACM SIGMOD, pages 1-12, 2000.

[12] X. He, R.S. Zemel, and M.A. Carreira-Perpinan. Multiscale conditional random fields for image labeling. In Proc. IEEE CVPR, pages 695-702, 2004.

[13] J. Lafferty, A. McCallum, and F. Pereira. Conditional random fields: probabilistic models for segmenting and labeling sequence data. In Proc. ICML, pages 282-289, 2001.

[14] W. Li, J. Han, and J. Pei. Accurate and efficient classifications based on multiple class-association rules. In Proc. IEEE ICDM, pages 369-376, 2001.

[15] D. Liu and J. Nocedal. On the limited memory BFGS method for large-scale optimization. Mathematical Programming, 45:503-528, 1989.

[16] B. Liu, W. Hsu, and Y. Ma. Integrating classification and association rule mining. In Proc. ACM SIGKDD, pages 80-86, 1998.

[17] R. Malouf. A comparison of algorithms for maximum entropy parameter estimation. In Proc. CoNLL, 2002.

[18] A. McCallum, D. Freitag, and F. Pereira. Maximum entropy markov models for information extraction and segmentation. In Proc. ICML, pages 591-598, 2000.

[19] A. McCallum. Efficently inducing features of conditional random fields. In Proc. UAI, 2003.

[20] F. Peng, F. Feng, and A. McCallum. Chinese segmentation and new word detection using conditional random fields. In Proc. COLING, 2004.

[21] S.D. Pietra, V.D. Pietra, and J. Lafferty. Inducing features of random fields. IEEE Trans. on Pattern Analysis and Machine Intelligence, 19(4):380-393, 1997.

[22] D. Pinto, A. McCallum, X. Wei, and W.B. Croft. Table extraction using conditional random fields. In Proc. ACM SIGIR, 2003.

[23] L.R. Rabiner. A tutorial on hidden markov models and selected applications in speech recognition. In Proc. the IEEE, $77(2): 257-286,1989$.

[24] A. Ratnaparkhi. A maximum entropy model for part-of-speech tagging. In Proc. EMNLP, 1996.

[25] F. Sha and F. Pereira. Shallow parsing with conditional random fields. In Proc. HLT/NAACL, 2003.

[26] A. Torralba, K.P. Murphy, and W.T. Freeman. Contextual models for object detection using boosted random fields. In Proc. NIPS, 2004.

[27] G. Yeo and C.B. Burge. Maximum entropy modeling of short sequence motifs with applications to RNA splicing signals. In Proc. Conf. on Computational Molecular Biology, pages 322-331, 2003 